\title{
Leukocyte-Reactive Antibodies in Female Blood Donors: The Austrian Experience
}

\author{
Marlies Schönbacher ${ }^{a} \quad$ Nicole Aichinger ${ }^{c}$ Lisa Weidner ${ }^{b} \quad$ Christof Jungbauer $^{b}$ \\ Christoph Grabmer ${ }^{c}$ Beate Schuha ${ }^{c}$ Eva Rohde ${ }^{c}$ Wolfgang Mayr ${ }^{a}$ b \\ Günther Körmöczi ${ }^{a}$ \\ ${ }^{a}$ Department of Blood Group Serology and Transfusion Medicine, Medical University of Vienna, Vienna, Austria; \\ ${ }^{\mathrm{b}}$ Austrian Red Cross, Blood Service for Vienna, Lower Austria and Burgenland, Vienna, Austria; ${ }^{\mathrm{C}}$ Department of \\ Transfusion Medicine, University Hospital Salzburg, Salzburg, Austria
}

\section{Keywords}

Human neutrophil antibodies · Human leukocyte antibodies · Female blood donors

\begin{abstract}
Introduction: Antibody-mediated transfusion-related acute lung injury (TRALI) is caused by antibodies against human leukocyte antigens (HLAs) or human neutrophil antigens (HNAs), and is one of the most serious complications associated with transfusion medicine. Prevention strategies like testing allo-exposed female blood donors have not yet been introduced nationwide in Austria. To assess the need and feasibility of routine leukocyte antibody testing, the prevalence of leukocyte-reactive antibodies in an Austrian female donor population was been determined using classical cellbased methods which were compared with a high-throughput bead-based method. Methods: Sera from 1,022 female blood donors were screened using a granulocyte aggregation test (GAT) and a white blood cell immunofluorescence test (WIFT) after retesting and specification of positive samples by granulocyte immunofluorescence test (GIFT) and monoclonal antibody-specific immobilization of granulocyte antigens (MAIGA). Potential HLA reactivities were confirmed using the microbeads assay LabScreen ${ }^{\top \mathrm{M}}$ Mixed. The results in 142 donor sera and 38 well-defined reference sera were investigated by the microbeads assay LabScreen ${ }^{\text {TM }}$
\end{abstract}

Multi and compared with classical cell-based methods. $\boldsymbol{R e}$ sults: Reactivity with either granulocytes and/or lymphocytes was detected in 79 sera (7.7\%), with the majority being HLA-specific. Antibodies against HNA were obtained in 7 samples $(0.7 \%)$. The aggregating potential of the detected antibodies was observed in 9 cases $(0.9 \%)$. Most of the leukocyte-reactive antibodies occurred at a donor age of between 35 and 59 years $(n=61)$. LabScreen Multi showed good agreement ( $\mathrm{k}=0.767$ ) for HNA antibody detection by cell-based assays, but double/multiple specificities (100\% of 7 anti-HNA- $1 \mathrm{~b}$ sera) as well as false-negative results ( $40 \%$ of 15 HNA-3-specific sera) occurred. Discussion: Leukocyte-reactive antibody screening is advised in Austrian female donors for safe blood transfusion, including single-donor convalescent plasma treatment of COVID-19 that may be implemented soon. For the introduction of LabScreen Multi, the combination with GAT should be considered to ensure correct anti-HNA-3a detection.

๑) 2020 S. Karger AG, Basel

\section{Introduction}

Transfusion-related acute lung injury (TRALI) is one of the most serious complications of transfusion and a leading cause of transfusion-related morbidity and mortality worldwide. About $80 \%$ of all TRALI cases are asso- 
ciated with leukocyte-reactive antibodies directed against either human leukocyte antigens (HLAs) or human neutrophil antigens (HNAs), which have the potential to activate primed neutrophils, monocytes, and/or pulmonary endothelial cells, resulting in capillary leakage and lung edema [1]. The pathophysiology of TRALI is, to date, incompletely understood. A two-hit model was hypothesized describing the clinical condition of the patient (e.g., inflammation) as the first hit, and blood-product factors (e.g., leukocyte-reactive antibodies and bioactive lipids) as the second hit, both necessary to induce TRALI, a lifethreatening syndrome of acute respiratory distress for which no treatments are available [1-5].

The formation of leukocyte-reactive antibodies is most probable in women with a history of pregnancy due to the risk of immunization against fetal HLA or HNA of paternal origin. Thus, plasma-containing blood products like fresh-frozen plasma (FFP) or platelet concentrates from female blood donors represent the highest risk factor for antibody-mediated TRALI. Aggregating antibodies like anti-HNA-3a, in particular, have the potential to induce possibly fatal TRALI, even when only a small amount of plasma, like that in red blood cell (RBC) concentrates, is transfused [6].

For many years, in Austria, the pooling of plasma has been standard for the production of solvent/detergent (S/D) FFP, which has led to the dilution of potential leukocyte-reactive antibodies with the plasma from other donors. This approach might explain why reports of TRALI cases caused by S/D FFPs are rare. Nevertheless, based on standard definitions and classifications from the International Society of Blood Transfusion (ISBT) and the International Hemovigilance Network (IHN), the Austrian Bundesamt für Sicherheit im Gesundheitswesen (BASG) publishes 1-3 TRALI cases per year, mainly associated with RBCs and platelets [7]. Most of them get classified as possible or probable TRALI, based on the clinical symptoms of the patients. In 2009, 2 TRALI cases ( 1 fatal) were declared as confirmed. Retrospective leukocyte antibody testing was rarely performed, relying on TRALI as a clinically defined syndrome, even without anti-HLA or antiHNA antibody detection [7]. In clinical practice, it may be difficult to differentiate TRALI from transfusion-associated circulatory overload (TACO) or transfusion-associated dyspnea (TAD), so one can only speculate on the number of TRALI cases that went unreported $[1,8,9]$.

Despite the use of S/D FFP in Austria, TRALI may now get more attention because, in the current COVID-19 pandemic, convalescent plasma of single donors might be widely used to treat severe acute respiratory syndrome coronavirus 2 (SARS-CoV-2) infection $[10,11]$. Such therapy was beneficial in treating related coronavirus-induced etiologies, such as Middle East respiratory syndrome coronavirus (MERS-CoV) infection and SARS [12]. In the ab- sence of both a specific antiviral treatment and vaccine, plasma transfusions containing neutralizing antibodies against SARS-CoV-2 are a promising passive immunization option for COVID-19 patients [13, 14]. Interestingly, TRALI and COVID-19 share similarities in the proinflammatory respiratory scenario, e.g., neutrophil priming, elevated cytokine levels, and the formation of neutrophil extracellular traps (NETs) indicate that neutrophils might play a key role in both TRALI and COVID-19 [1, 15-17]. As the inflammatory state of severe and critically ill COVID-19 patients may pose a particular risk for the development of single-donor plasma-induced TRALI in this fragile cohort, TRALI prevention by leukocyte antibody testing of convalescent single-donor plasma appears essential.

The allo-immunization rate against leukocyte antigens in Austrian female blood donors is unknown. Moreover, Austria has not yet introduced nationwide TRALI prevention strategies like leukocyte antibody screening of allo-exposed female donors versus blood centers in Germany, France, the UK, Sweden, and parts of the USA routinely screen selected donor cohorts, at least for antibodies against HLA using ELISA and/or a microbeads assay [18]. One reason for this might be that HNA antibody testing, in particular, represents a major hurdle, as standard cell-based methods need freshly isolated granulocytes, are technically demanding, and are not suitable for high throughput testing. The logistics and financial complexity of implementation represent further hindrances.

This study was designed to assess the need and feasibility of routine leukocyte antibody testing. In addition to determining the prevalence of leukocyte-reactive antibodies in the Austrian female donor population, the standard cell-based methods are compared with a high throughput bead-based method.

\section{Materials and Methods}

Three Austrian donor services participated in this study, namely, the Medical Universities of Vienna and Salzburg and the Austrian Red Cross. They all provided sera from female blood donors who were not pregnant within the 6 months before donating blood.

For cell-based antibody screening, a panel of 3 HNA-typed, freshly isolated white blood cells (WBCs), expressing at least HNA-1a, HNA-1b, HNA-2, HNA-3a, HNA-3b, HNA-4a, and HNA-5a as well as HLA-A2 was used. Granulocytes were isolated with the EasySep ${ }^{\mathrm{TM}}$ direct neutrophil isolation kit (Stemcell Technologies, Cologne, Germany). Whole leukocytes were isolated by dextran sedimentation following ammonium chloride lysis of residual red cells. Cells were adjusted at $5 \times 10^{3}$ leukocytes $/ \mu \mathrm{L}$ for all screening tests.

\section{Cell-Based Antibody Detection}

All sera underwent leukocyte antibody screening with a microscopic granulocyte aggregation test (GAT) and a flow-cytometric WBC immunofluorescence test (WIFT), as described [19-21]. 
In brief, for GAT, isolated granulocytes were incubated with serum in Terasaki plates for $2 \mathrm{~h}$ at $37^{\circ} \mathrm{C}$ and checked for cell aggregation under light microscopy. GAT is very specific for finding granulocyte-aggregating antibodies against HNA and HLA class I. Anti-HNA-3 antibodies, in particular, are best detected in GAT, because of their unique aggregation pattern. Other anti-HNA/ HLA antibodies can induce aggregation, but not dependably [20, 22].

For WIFT, whole leukocytes were incubated with sera for 30 $\min$ at $37^{\circ} \mathrm{C}$. After 2 washing steps, secondary antibody Alexa Fluor 488 goat anti-human IgG (Jackson ImmunoResearch, Newmarket, UK) was added and incubated in the dark at room temperature for $30 \mathrm{~min}$. After 1 further washing step, cells were resuspended in Cellfix ${ }^{\mathrm{TM}}$ (Becton Dickinson, Heidelberg, Germany) before analysis. Flow cytometry was performed on a FACSCanto II with FACS Diva software (BD Biosciences). Leukocytes were gated by forward and side scatter, and fluorescence intensity was detected for each leukocyte subset (granulocytes, monocytes, and lymphocytes). Results were defined as positive when the ratio of median fluorescence intensity (MFI) between sample and negative control exceeded 3, based on our own experience in routine neutrophil antibody diagnostics. WIFT is able to detect and differentiate antibodies against HNA and HLA, by evaluating panel- and leukocyte subset-specific reactivity.

Based on panel reactivity, granulocyte-reactive sera were further tested with paraformaldehyde-fixed neutrophils using the microscopic granulocyte immunofluorescence test (GIFT) and the monoclonal antibody-specific immobilization of granulocyte antigens assay (MAIGA) [23,24]. GIFT was used as a screening assay, and, for antibodies which could not be immobilized in MAIGA like anti-HNA-3a, as a specification test. Briefly, GIFT was performed by incubating sera and neutrophils for $30 \mathrm{~min}$ at $37^{\circ} \mathrm{C}$. After 3 washing steps, rabbit anti-Human IgG (Dako, Glostrup, Denmark) was added and incubated in the dark at room temperature for $30 \mathrm{~min}$. After 3 further washing steps, cell-bound fluorescence was assessed by fluorescence microscopy.

For MAIGA, sera were incubated with $10 \times 10^{3}$ leukocytes/ $\mu \mathrm{L}$ for $30 \mathrm{~min}$ at $37^{\circ} \mathrm{C}$. After washing, the following mouse monoclonal antibodies were added and incubated for $30 \mathrm{~min}$ at $37^{\circ} \mathrm{C}$ : DJ130c (Acris, Herford, Germany), 3G8 (Beckman Coulter, Krefeld, Germany) and LNK-16 (GeneTex, Irvine, CA, USA) against CD16 (HNA-1); MEM-166 (Acris) for NB-1 (HNA-2), Bear-1 (Beckman Coulter) for CD11b (HNA-4), 7E4 (Beckman Coulter) for CD18 (HNA-4 \& -5), B1G6 (Beckman Coulter) for $\beta 2$-microglobulin (part of HLA Cl I), and W6/32 (eBioscience, San Diego, CA, USA) for HLA Cl I. For the HNA-3 bearing glycoprotein CTL-2, there is still no monoclonal antibody available [22]. After cell lysis with Pefabloc ${ }^{\circledR}$ SC and Leupeptin Hemisulfate (Sigma-Aldrich, St. Louis, USA), trimolecular antigen-antibody-complexes were transferred to an anti-mouse IgG-coated ELISA plate and detected with anti-human IgG/HRP following substrate addition (O-phenylene-diamine-hydrochloride; Sigma-Aldrich). Optical density (OD) was analyzed by a microplate reader (Phomo, Anthos Mikrosysteme). Despite MAIGA being able to differentiate between anti-HNA and anti-HLA class I, detected anti-HLA reactivities were not considered in the final results because HLA antibody detection was performed as mentioned below.

\section{Bead-Based Antibody Detection}

LabScreen ${ }^{\mathrm{TM}}$ Mixed

All positively screened samples were tested for anti-HLA class I/II antibodies using the microbeads assay, LabScreen Mixed (One Lambda, Canoga Park, CA, USA) according to the manufacturer's description, with a cut-off at a normalized background (NBG) ratio $\geq 5.0$.

Leukocyte-Antibodies in Austrian Female Blood Donors

\section{LabScreen $^{\mathrm{TM}}$ Multi}

Donor sera from the Medical University Salzburg were tested using LabScreen Multi (One Lambda), a bead-based assay specifying antibodies against HNA-1a/b/c, HNA-2, HNA-3a/b, HNA-4a, and HNA-5a/b as well as differentiating antibodies against HLA classes I and II. This kit works with color-coded microbeads, each bead population being coated with either HLA class I/II peptides or single HNA peptides, respectively. Besides a routinely used negative control serum (One Lambda) to calculate the NBG ratio, well-defined reference samples with or without known HNA antibodies were included. These reference sera were evaluated using GAT, GIFT, and WIFT prior to method comparison. After incubation of beads with sera, bound antibodies were detected with $\mathrm{R}$ phycoerythrin (PE)-conjugated anti-Human IgG (One Lambda). Fluorescence was detected with a Luminex flow analyzer and evaluated with HLA Fusion ${ }^{\mathrm{TM}}$ software (One Lambda). Cut-off values were set as positive at an NBG ratio of $\geq 6$ for microbeads specific to HLA, $\geq 5$ for HNA-1, $\geq 30$ for HNA-2, and $\geq 10$ for HNA-3, HNA-4, and HNA-5, based on the study of Schulz et al. [25] and previous validation. After testing with LabScreen Multi, all sera underwent cell-based antibody testing, as mentioned above.

For comparison with the results of cell-based methods, antiHLA antibodies were excluded, as bead-based HLA diagnostics has been well-established and approved for many years. Hence, method comparison focused exclusively on the detection of HNA antibodies, where cell-based methods were considered as the gold standard for method comparison.

\section{HNA Typing}

From donors with identified anti-HNA antibodies, DNA was isolated from EDTA whole blood using the $\mathrm{EZ} 1{ }^{\circledR}$ DNA Blood 350 $\mu \mathrm{L}$ kit (Qiagen, Hilden, Germany) and typed for HNA-1a/b/c, HNA-3a/b, HNA-4a/b, and HNA-5a/b by commercially available PCR-SSP (HNA Ready Gene, Inno-train, Kronberg, Germany) to confirm antigen specificity. Serologic HNA-2 typing was performed by flow cytometry, similar to WIFT, using a reference serum containing anti-HNA-2 antibodies. Granulocytes from a healthy donor expressing HNA-2 served as a positive control.

\section{Statistics}

Statistics were performed using SPSS v24 software (Armonk, NY, USA). Prevalence of granulocyte-reactive antibodies was calculated including estimated $95 \%$ confidence intervals (CIs). Cohen's $\kappa$ was used for the comparison of cell-based and bead-based methods.

\section{Results}

A study flow chart including all samples, methods and general results is depicted in Figure 1.

\section{Antibody Testing of Female Blood Donors}

Overall, sera from 1,022 female blood donors underwent GAT and WIFT. Of these, 79 samples (7.7\%) were reactive with granulocytes and/or lymphocytes, including $9(0.9 \%)$ with aggregating potential as judged from the positive GAT results (Table 1 ).

Antibodies against HLA class I were detected in 37 samples (3.6\%), while mixed antibodies against HLA classes I and II were found in 34 sera $(3.3 \%, 1$ serum ad- 


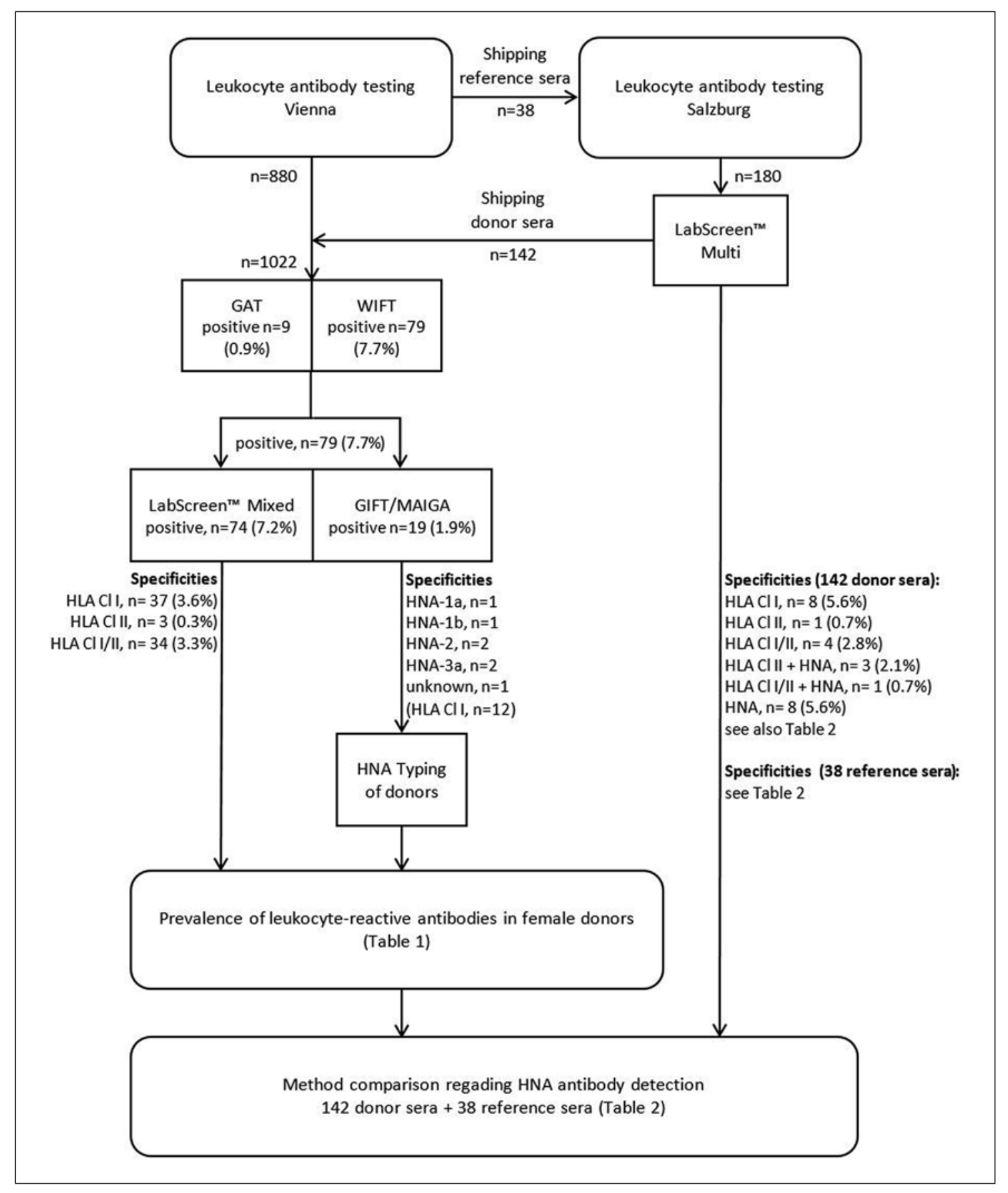

Fig. 1. Flow chart depicting all relevant works and results of the study.

ditionally specific for HNA-2). Antibodies against HLA class II were detectable in 3 cases $(0.3 \%, 1$ serum also containing anti-HNA-1b, as described below).

Seven donors (0.7\%) had neutrophil-specific antibodies. Six donors showed antibodies specific for HNA: antiHNA-1a $(n=1)$, anti-HNA-1b $(n=1)$, anti-HNA-2 $(n=$ $2)$, and anti-HNA-3a $(n=2)$. One donor had granulocytespecific antibodies of unknown HNA specificity, reactive in GIFT and WIFT, but negative in GAT and MAIGA, even after remeasurement. LabScreen Mixed was nega- tive but LabScreen Multi detected antibodies against HNA-4a (and HLA class II, but weakly above the cut-off), as mentioned below. Genotyping of this donor could not be performed due to the lack of DNA.

All anti-HNA-3a as well as anti-HNA-1a and antiHNA- 1 b antibodies could be confirmed as allo-immune by PCR-SSP, as these donors did not carry the respective antigen. One of 2 donors with anti-HNA- 2 was serologically typed as HNA-2-negative (HNA- $2_{\text {null }}$ ). The second donor could not be typed for HNA-2 because no freshly 
Fig. 2. Histogram depicting the age distribution of female blood donors. Donors with positive cell-based antibody screening (red bars) are overlaid on all donors (grey bars).

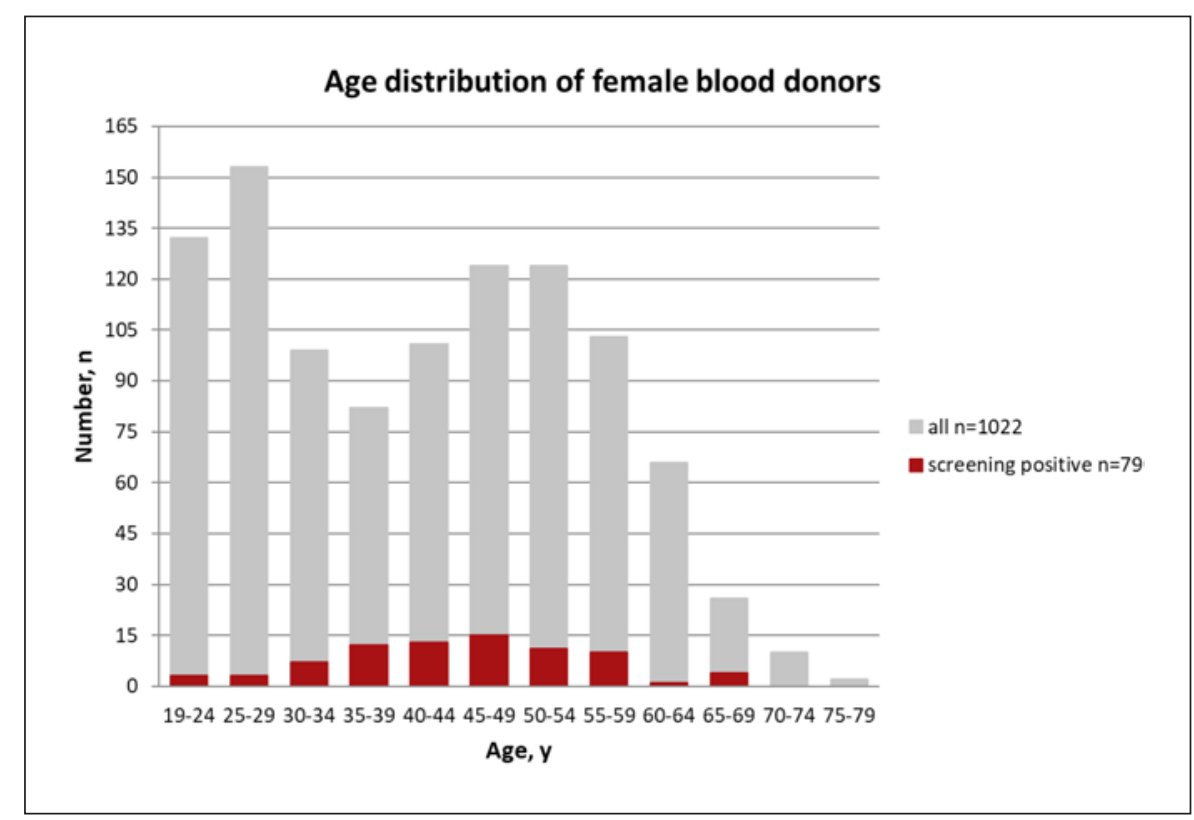

Table 1. Contingency table of positively tested sera from 1,022 female donors

\begin{tabular}{lllll}
\hline \multicolumn{5}{l}{ Cell-based leukocyte antibody screening } \\
\cline { 2 - 5 } & $\begin{array}{l}\text { granulocyte- } \\
\text { reactive, } n\end{array}$ & $\begin{array}{l}\text { lymphocyte- } \\
\text { reactive, } n\end{array}$ & $\begin{array}{l}\text { leukocyte-reactive, } \\
n \text { (aggregating) }\end{array}$ & total, $n(\%)$ \\
\hline $\begin{array}{l}\text { Specificity } \\
\text { HLA class I }\end{array}$ & 1 & & & \\
HLA class II & & 34 & 1 & $37(3.6)$ \\
HLA class I/II & 3 & 20 & $13(7)$ & $2(0.2)$ \\
HNA & 1 & $2(2)$ & $5(0.5)$ \\
HNA + HLA class II & 1 & & $1(0.1)$ \\
HNA + HLA class I/II & 1 & 55 & $18(9)$ & $79(7.7[95 \%$ CI 6.1-9.4]) \\
\hline Total & 6 & & & $1(0.1)$ \\
\hline
\end{tabular}

HLA, human leukocyte antigen; HNA, human neutrophil antigen; CI, confidence interval.

isolated neutrophils were available. Unfortunately, novel HNA-2 genotyping according to Bayat et al. [26] has not yet been implemented. However, both GIFT and WIFT showed a mixed pattern of fluorescent and nonfluorescent granulocytes, which is distinctive for antibodies against HNA-2 [27, 28]. Furthermore, MAIGA was positive with MEM-166, a monoclonal antibody specific for the HNA-2-bearing glycoprotein CD177, thereby confirming the screening results. Additionally, LabScreen Multi was able to detect anti-HNA-2 antibodies in this donor and in the HNA-2 $2_{\text {null }}$ donor, as described below. Hence, HNA-2 specificity can be demonstrated by 4 different methods. This antibody is probably of allo-immune nature as autoimmune anti-HNA-2 might be exceedingly rare in healthy blood donors [22].

The age distribution of all female blood donors is depicted in Figure 2. Most donors were between the age of
40 and 59 years $(n=452)$, followed by a cohort aged $19-29$ years $(n=285)$. More than three-quarters of positive screening results were obtained in the age group of 35-59 years $(n=61)$, and peaked at $45-49$ years $(n=15)$.

\section{Comparison of Cell-Based and Bead-Based Methods}

Regarding HNA Specification

LabScreen Multi was deployed to investigate sera from 142 donors as well as 38 reference sera. Bead-based HNA specificity results were compared with those obtained by cell-based methods (Table 2). HLA reactivity was not considered in this comparison, even though it is mentioned in Figure 1. The most important data (NBG ratios of HNA specificity, false-positive, and false-negative results) obtained by LabScreen Multi are shown in Table 3.

Independent of specificity, both the cell-based and bead-based methods detected anti-HNA antibodies in 31 
Table 2. Results in donor and reference samples regarding HNA antibody specification

\begin{tabular}{|c|c|c|c|c|c|c|c|c|c|c|}
\hline & \multicolumn{10}{|c|}{ Cell-based HNA antibody specification } \\
\hline & negative & 1a & $1 \mathrm{~b}$ & $1 \mathrm{c}$ & 2 & $3 a$ & $3 b$ & $4 \mathrm{a}$ & unknown & total \\
\hline \multicolumn{11}{|c|}{ Bead-based HNA antibody specification ${ }^{1}$} \\
\hline \multicolumn{11}{|c|}{ Donors } \\
\hline Negative & 130 & & & & & & & & & 130 \\
\hline la & 3 & & & & & & & & & 3 \\
\hline 2 & 1 & & & & 2 & & & & & 3 \\
\hline $3 a$ & 3 & & & & & 1 & & & & 4 \\
\hline $4 \mathrm{a}$ & 1 & & & & & & & & 1 & 2 \\
\hline All & 138 & & & & 2 & 1 & & & 1 & 142 \\
\hline \multicolumn{11}{|l|}{ References } \\
\hline Negative & 5 & & & & & 4 & 2 & & & 11 \\
\hline 1a & & 4 & & & & & & & & 4 \\
\hline $1 b+1 c$ & & & 6 & & & & & & & 6 \\
\hline $1 b+1 c+2$ & & & 1 & & & & & & & 1 \\
\hline $1 \mathrm{c}$ & & & & 1 & & & & & & 1 \\
\hline 2 & & & & & 4 & & & & & 4 \\
\hline $1 c+2$ & & & & & 1 & & & & & 1 \\
\hline $3 a$ & & & & & & 3 & & & & 3 \\
\hline $3 b$ & & & & & & & 2 & & & 2 \\
\hline $3 a+3 b$ & & & & & & 4 & & & & 4 \\
\hline $4 \mathrm{a}$ & & & & & & & & 1 & & 1 \\
\hline All & 5 & 4 & 7 & 1 & 5 & 11 & 4 & 1 & & 38 \\
\hline Total & 143 & 4 & 7 & 1 & 7 & 12 & 4 & 1 & 1 & 180 \\
\hline
\end{tabular}

of 180 samples (donor and reference sera). Using LabScreen Multi, 6 reference samples containing HNA antibodies were negative, but 8 additional donor sera tested positive for HNA antibodies (listed in more detail below). Negative results were obtained in 135 samples with both methods. Cohen's $\kappa$ was 0.767 , indicating a good agreement between the detection methods.

Of 142 donor sera, 134 (94.4\%) were congruent with both methods, 130 (91.6\%) of which tested negative and 4 of which were positive. Cell-based methods revealed 2 anti-HNA-2-specific and 1 anti-HNA-3a-specific samples, confirmed with LabScreen Multi (2.1\%). One further sample $(0.7 \%)$ was specific for HNA-4a and HLA class II in LabScreen Multi and also reactive with granulocytes in GIFT and WIFT, but negative in GAT and MAIGA. In 8 sera (5.6\%), HNA-specific antibodies were detected using LabScreen Multi, namely, anti-HNA-1a ( $n$ $=3)$, anti-HNA-2 $(n=1)$, anti-HNA-3a $(n=3)$, and antiHNA-4a $(n=1)$, but the cell-based methods did not yield any reactivity.

From 38 reference sera, 5 were found to be without HNA antibodies. The other 33 contained HNA antibodies with the following specificities: HNA-1a $(n=4)$, HNA$1 \mathrm{~b}(n=7)$, HNA-1c $(n=1)$, HNA-2 $(n=5)$, HNA-3a $(n$ $=11)$, HNA-3b $(n=4)$, and HNA-4a $(n=1)$. Thirty-two samples were congruent in both methods (all 5 negative controls were negative and 27 sera containing anti-HNA antibodies were positive). Identical specificities were obtained in 20 cases (52.6\%) including all anti-HNA-1a-, anti-HNA-1c-, and anti-HNA-4a-containing sera. With LabScreen Multi, multiple specificities were obtained in 12 samples (31.6\%), including all 7 containing anti-HNA$1 \mathrm{~b}$, specified as combined anti-HNA-1b/1c in 6 cases and anti-HNA-1b/2 in 1 case (Table 3 ). One anti-HNA-2 serum was additionally positive with the HNA-1c beads. Four anti-HNA-3a sera were reactive with HNA-3a and HNA-3b beads in LabScreen Multi. Six HNA-3-specific sera (15.8\%), i.e., 4 containing anti-HNA-3a and 2 containing anti-HNA-3b, could not be detected by LabScreen Multi.

\section{Discussion}

Screening of allo-exposed blood donors for the presence of leukocyte-reactive antibodies (against HLA and/ or HNA) has not yet been introduced in Austria to date. The reasons for this could be the ill-defined epidemiology of antileukocyte allo-immunization of the Austrian donor population, the logistics and financial complexity of 
Table 3. All HNA specificities and missing specificities obtained by bead-based LabScreen Multi

\begin{tabular}{|c|c|c|c|c|c|c|c|c|c|}
\hline \multirow{2}{*}{$\begin{array}{l}\text { Sample } \\
\text { No. }\end{array}$} & \multirow[t]{2}{*}{ Sample } & \multirow{2}{*}{$\begin{array}{l}\text { HNA } \\
\text { specificity }\end{array}$} & \multicolumn{7}{|c|}{ NBG ratio per bead } \\
\hline & & & 1a & $1 b$ & $1 c$ & 2 & $3 a$ & $3 b$ & $4 a$ \\
\hline $1-4$ & references & 1a & $42.2-60.5$ & & & & & & \\
\hline $5-10$ & references & $1 \mathrm{~b}$ & & $36.7-180.0$ & $22.9-202.0$ & & & & \\
\hline 11 & reference & $1 b$ & & 36.7 & 31.0 & 30.5 & & & \\
\hline 12 & reference & $1 \mathrm{c}$ & & & 135.9 & & & & \\
\hline 13 & reference & 2 & & & 202.0 & 274.5 & & & \\
\hline $14-17$ & references & 2 & & & & $66.3-407.0$ & & & \\
\hline $18-21$ & references & $3 a$ & & & & & $186.2-350.4$ & $15.0-111.1$ & \\
\hline $22-25$ & references & $3 a$ & & & & & $<5.0$ & & \\
\hline $26-28$ & references & $3 a$ & & & & & $57.9-149.9$ & & \\
\hline 29,30 & references & $3 b$ & & & & & & $<5.0$ & \\
\hline 31,32 & references & $3 b$ & & & & & & $15.7-58.8$ & \\
\hline 33 & reference & $4 \mathrm{a}$ & & & & & & & 10.0 \\
\hline $34-36$ & donors & none & $6.0-37.7$ & & & & & & \\
\hline 37,38 & donors & 2 & & & & $188.3-317.0$ & & & \\
\hline 39 & donor & none & & & & 48.4 & & & \\
\hline 40 & donor & $3 a$ & & & & & 22.2 & & \\
\hline 41 & donor & HLA class I & & & & & 33.8 & & \\
\hline 42,43 & donors & none & & & & & $16.8-16.9$ & & \\
\hline 44 & donor & none & & & & & & & 49.4 \\
\hline 45 & donor & unknown & & & & & & & 40.7 \\
\hline
\end{tabular}

implementation, and the routine use of $\mathrm{S} / \mathrm{D}$ pooled plasma. Nevertheless, TRALI is still associated with RBCs and platelets, indicating that leukocyte antibody-containing plasma residuals of $<20 \mathrm{~mL}$ appear sufficient to induce TRALI $[6,9,29]$.

The current COVID-19 pandemic may further emphasize the relevance of TRALI prevention by leukocyte antibody testing, as convalescent single-donor plasma transfusion for the treatment of COVID-19 [30] may pose a particular risk, especially when plasma from female donors with a history of pregnancy is administered.

Our study shows that Austrian female blood donors have an allo-immunization rate of 7.7\% (95\% CI 6.19.4\%) against leukocyte antigens. This prevalence is comparable with the study of Reil et al. [6] who revealed an allo-immunization rate in parous female donors of $8.9 \%$ when using similar techniques. Unfortunately, in our study, the number of previous pregnancies could not be evaluated. Before and within the period of sample collection, only pregnancies within 6 months before blood donation were considered in the blood donor questionnaire. Especially in view of the unselected female donor cohort analyzed in this study, presumably including many women with no history of allo-exposure, the antibody prevalence that was detected seems considerable. Since 2018, a new donor questionnaire has been introduced in which a complete history of pregnancy must be recorded. Hence, female blood donors can now be recognized as potentially allo-immunized and therefore considered for future antibody screening. The donors at the highest risk for having anti-leukocyte antibodies were 35-59 years of age, indicating that this population might most likely have a history of pregnancy. It is well-known that the number of pregnancies is a risk factor for developing leukocyte antibodies $[6,31,32]$. Unsurprisingly, antibody prevalence decreased at $\geq 60$ years of age, most probably because antibodies became undetectable decades after pregnancy [32].

In contrast, Nguyen et al. [33] reported an allo-immunization rate against HLA/HNA in female parous blood donors of about $22 \%$, with the proportion of HNA antibodies amounting to $1.5 \%$. The lower antibody prevalence revealed in our study might have been due to the fact that we did not conduct classic HLA antibody testing (i.e., the microbeads assay) on all sera, only those which were drawn at the Medical University of Salzburg, or when cell-based testing at the Medical University of Vienna was positive, respectively. The cell panel did not cover all HLAs, besides the fact that only a subset of mononuclear cells analyzed in WIFT expresses HLA class II antigens. Some of anti-HLA class I/ II might thus go undetected in WIFT, and the prevalence of HLA antibodies in Austrian female blood donors is probably even higher. For future antibody screening using WIFT, an additional staining of mononuclear cells with anti-CD19 or -CD20 might be useful to allow reliable detection of antiHLA class II, when standard HLA antibody detection is not performed. Since the majority of TRALI cases are 
caused by anti-HLA antibodies [2, 6, 34, 35], even though anti-HNA antibodies cause more fatalities [6], introducing leukocyte antibody testing of blood donors should include classic detection methods for anti-HLA class I/II and anti-HNA antibodies.

Another important finding is that about $1 \%$ of all tested samples were positive in GAT and therefore contained antibodies with granulocyte-aggregating potential, formerly known as "leucoagglutinins". These antibodies, particularly anti-HNA-3a, are frequently associated with severe and fatal TRALI because of their potential to induce the active aggregation of neutrophils $[2,6,36,37]$. Not all transfusions containing such antibodies will lead to full-blown TRALI because of dilution during the preparation of blood products (pooled plasma and additive solution in platelet concentrates) or in the patient (e.g., infusions). Other protective factors include compatibility with the recipient's antigens and the absence of systemic inflammation, trauma, and mechanical ventilation that lead to neutrophil priming, as postulated in the two-hitmodel [1-3]. Some patients may develop only transfusion-associated neutropenia (TRAIN) without pulmonary complications $[38,39]$. Nevertheless, TRALI is likely underdiagnosed in clinical practice because of the symptomatic overlap with a wealth of alternative pulmonary disorders including TACO [1].

Antibody screening of female blood donors has recently been introduced at the University Hospital Salzburg, where the LabScreen Multi, a commercial microbeads assay to detect antibodies against HLA class I, HNA class II, and HNA $1-5$, is performed. This assay is easy to handle and is suitable for high-throughput-screening, but is also cost-intensive and not considered to be the gold standard for the detection of anti-HNA antibodies [25, 40]. The test is also not ideal for the detection of antiHNA-3a, which is known as the most dangerous antibody to induce TRALI.

In 2017, Schulz et al. [25] evaluated the LabScreen Multi; $10 \%$ of HNA-antibodies were false-negative and $5.5 \%$ of negative controls were false-positive. Most of the falsenegative results occurred with HNA-3-specific sera (4/39 anti-HNA-3a and 4/ 6 anti-HNA-3b sera). In our study, the false-negative rate was $18 \%$, i.e., it was detected in $6 / 33$ anti-HNA reference sera (all with HNA-3 specificity), but none of the 5 negative reference sera was false-positive. In other words, an alarming rate of $40 \%$ (6/15 samples) of reference sera containing anti-HNA-3 antibodies were not detected by LabScreen Multi, possibly due to conformational changes of the recombinant protein during the coating process on the bead. It remains unclear why the falsenegative rate in our study was higher than in the study of Schulz et al. [25]. Even the lot numbers of the test kit used in the studies were different, so no significant changes were made on behalf of the manufacturer.
Considering the 142 donor sera, however, false-positive reactions occurred in 5.6\% (8 cases) which seems similar but not comparable to the false-positive rate of Schulz et al. [25] (5.5\%), as donor sera did not serve as reference samples in their study.

Furthermore, similar to the study of Schulz et al. [25] in $100 \%$ of anti-HNA-1b sera and $33 \%$ (4/12 samples, including 1 donor and 11 reference sera) of anti-HNA-3a sera, double specificity (positivity with HNA-1b and HNA-1 1 or HNA-3a and HNA-3b beads) were observed, even though these sera had clear-cut monospecific reactions in the cell-based methods. The HNA-1b and HNA$1 \mathrm{c}$ beads were coated with the protein which is encoded by FCGR $3 B^{*} 03$, bearing both epitopes. Therefore, double specificity within the HNA-1 group are not surprising. The reason for false-negative reactions as well as doublepositive reactions with both HNA-3a and HNA-3b beads might be the loss of the natural conformation of the recombinant protein CTL2 during the coating process [ 25 , $27,36,37]$. One anti-HNA-1b serum was additionally weakly positive with the HNA-2 bead, and 1 HNA-2-specific serum was positive with the HNA-1b and HNA-1c bead. All of the double/multiple specificities, apart from HLA, could be confusing for unexperienced users. We showed that, in $10 / 12$ cases, the specificity with the highest NBG ratio complied with the true specificity (Table $3)$.

All 7 HNA-2-specific sera (2 donor and 5 reference sera) were correctly identified. Results that matched perfectly with cell-based assays were only obtained with the reference sera that contained anti-HNA-1a, anti-HNA$1 c$, and anti-HNA-4a.

Based on these findings, LabScreen Multi, at least, should be applied in combination with the GAT as a screening test, to ensure the reliable detection of antiHNA-3a [41]. The GAT is the simplest of all cell-based neutrophil antibody-detecting methods, requires only a small number of typed freshly isolated cells, and is known as the best method to identify anti-HNA-3a antibodies [37]. However, if possible, professional detection of anti-HNA antibodies using more time-consuming methods (GIFT, WIFT, and MAIGA) should be implemented.

In conclusion, bead-based methods for the detection of leukocyte antibodies are fast and sensitive screening tests but HNA antibody specificities are most reliably detected by classical cell-based methods. Based on the epidemiologic allo-immunization data, it is recommended that leukocyte antibody testing of blood donors at risk should be implemented in Austria to improve the quality of blood products beyond the use of S/D pooled plasma. The screening of female apheresis donors with a history of pregnancy would be a useful measure. 


\section{Statement of Ethics}

The study was approved by the Ethics Committee of the Medical University of Vienna (EK 1049/2017). All blood donors gave written informed consent for sample testing.

\section{Conflict of Interest Statement}

The authors have no conflicts of interest to declare.

\section{Author Contributions}

M.S. designed the study, analyzed the samples, evaluated and interpreted the data, and wrote the manuscript. C.J. and L.W. provided donor samples as well as important information about blood donation. N.A., E.R., C.G., and B.S. provided donor sera and analyzed samples using LabScreen ${ }^{\mathrm{TM}}$ Multi. W.R.M. interpreted data and assisted in statistical analysis. G.F.K. designed the study and interpreted the data. All authors critically revised and approved the manuscript.

\section{Funding Sources}

This study was funded by the Department of Blood Group Serology and Transfusion Medicine, Medical University of Vienna.

\section{References}

1 Semple JW, Rebetz J, Kapur R. Transfusionassociated circulatory overload and transfusion-related acute lung injury. Blood. 2019 Apr;133(17):1840-53.

2 Bux J. Antibody-mediated (immune) transfusion-related acute lung injury. Vox Sang. 2011 Jan;100(1):122-8

3 Toy P, Gajic O, Bacchetti P, Looney MR, Gropper MA, Hubmayr R, et al.; TRALI Study Group. Transfusion-related acute lung injury: incidence and risk factors. Blood. 2012 Feb; 119(7):1757-67.

4 van Stein D, Beckers EA, Sintnicolaas K, Porcelijn L, Danovic F, Wollersheim JA, et al. Transfusion-related acute lung injury reports in the Netherlands: an observational study. Transfusion. 2010 Jan;50(1):213-20.

5 Semple JW, McVey MJ, Kim M, Rebetz J, Kuebler WM, Kapur R. Targeting Transfusion-Related Acute Lung Injury: The Journey from Basic Science to Novel Therapies. Crit Care Med. 2018 May;46(5):e452-8.

6 Reil A, Keller-Stanislawski B, Günay S, Bux J. Specificities of leucocyte alloantibodies in transfusion-related acute lung injury and results of leucocyte antibody screening of blood donors. Vox Sang. 2008 Nov;95(4):313-7.

7 Gesundheitswesen B-BfSi. Hämovigilanzberichte 2009-2019. [updated 23.07.2019]. Available from: https://www.basg.gv.at/ news-center/publikationen/berichte/spezielle-berichte/.

8 Narayan S, Poles D. Serious Hazards of Transfusion (SHOT) Steering Group. The 2018 Annual SHOT Report 2019. Available from: https://www.shotuk.org/shot-reports/.

9 Andreu G, Boudjedir K, Muller JY, Pouchol E, Ozier Y, Fevre G, et al. Analysis of Transfusion-Related Acute Lung Injury and Possible Transfusion-Related Acute Lung Injury Reported to the French Hemovigilance Network From 2007 to 2013. Transfus Med Rev. 2018 Jan;32(1):16-27.

10 Investigational FD. COVID-19 Convalescent Plasma - Emergency INDs: U.S. Food and Drug Administration; 2020. [updated 26.03.2020]. Available from: https://www.fda. gov/vaccines-blood-biologics/investigational-new-drug-ind-or-device-exemption-ide- process-cber/investigational-covid-19-convalescent-plasma-emergency-inds.

11 Mehta P, McAuley DF, Brown M, Sanchez E, Tattersall RS, Manson JJ. COVID-19: consider cytokine storm syndromes and immunosuppression. Lancet (London, England). 2020.

12 Mair-Jenkins J, Saavedra-Campos M, Baillie JK, Cleary P, Khaw FM, Lim WS, et al.; Convalescent Plasma Study Group. The effectiveness of convalescent plasma and hyperimmune immunoglobulin for the treatment of severe acute respiratory infections of viral etiology: a systematic review and exploratory meta-analysis. J Infect Dis. 2015 Jan;211(1):80-90.

13 Cunningham AC, Goh HP, Koh D. Treatment of COVID-19: old tricks for new challenges. Crit Care. 2020 Mar;24(1):91.

14 Chen L, Xiong J, Bao L, Shi Y. Convalescent plasma as a potential therapy for COVID-19. Lancet Infect Dis. 2020 Apr;20(4):398-400.

15 Rebetz J, Semple JW, Kapur R. The Pathogenic Involvement of Neutrophils in Acute Respiratory Distress Syndrome and Transfusion-Related Acute Lung Injury. Transfus Med Hemother. 2018 Oct;45(5):290-8.

16 Barnes BJ, Adrover JM, Baxter-Stoltzfus A, Borczuk A, Cools-Lartigue J, Crawford JM, et al. Targeting potential drivers of COVID-19: neutrophil extracellular traps. J Exp Med. 2020 Jun;217(6):e20200652.

17 Huang C, Wang Y, Li X, Ren L, Zhao J, Hu Y, et al. Clinical features of patients infected with 2019 novel coronavirus in Wuhan, China. Lancet. 2020 Feb;395(10223):497-506.

18 Reesink HW, Lee J, Keller A, Dennington P, Pink J, Holdsworth R, et al. Measures to prevent transfusion-related acute lung injury (TRALI). Vox Sang. 2012 Oct;103(3):231-59.

19 Jiang AF, Lalezari P. A micro-technique for detection of leukocyte agglutinins. J Immunol Methods. 1975 Apr;7(1):103-8.

20 Nguyen XD, Flesch B, Sachs UJ, Kroll H, Klüter $\mathrm{H}$, Müller-Steinhardt M. Rapid screening of granulocyte antibodies with a novel assay: flow cytometric granulocyte immunofluorescence test. Transfusion. 2009 Dec;49(12):2700-8.

21 Heinzl MW, Schönbacher M, Dauber EM, Panzer S, Mayr WR, Körmöczi GF. Detection of granulocyte-reactive antibodies: a compar- ison of different methods. Vox Sang. 2015 Apr;108(3):287-93.

22 Flesch B. Work-up in the case of granulocyte antibodies. ISBT Sci Ser. 2020;15(1):59-69.

23 Verheugt FW, von dem Borne AE, Décary F, Engelfriet CP. The detection of granulocyte alloantibodies with an indirect immunofluorescence test. Br J Haematol. 1977 Aug;36(4): 533-44.

24 Bux J, Kober B, Kiefel V, Mueller-Eckhardt C. Analysis of granulocyte-reactive antibodies using an immunoassay based upon monoclonal-antibody-specific immobilization of granulocyte antigens. Transfus Med. 1993 Jun;3(2):157-62.

25 Schulz U, Reil A, Kiefel V, Bux J, Moog R. Evaluation of a new microbeads assay for granulocyte antibody detection. Transfusion. 2017 Jan;57(1):70-81.

26 Bayat B, Bein G, Sachs UJ. A sequence-specific polymerase chain reaction method for HNA-2 genotyping: homozygous c.843A $>$ T mutation predicts the absence of CD177. Transfusion. 2016 Aug;56(8):2127-32.

27 Bux J. Human neutrophil alloantigens. Vox Sang. 2008 May;94(4):277-85.

28 Flesch BK, Reil A. Molecular Genetics of the Human Neutrophil Antigens. Transfus Med Hemother. 2018 Oct;45(5):300-9.

29 Win N, Chapman CE, Bowles KM, Green A, Bradley S, Edmondson D, et al. How much residual plasma may cause TRALI? Transfus Med. 2008 Oct;18(5):276-80.

30 Liang T. Handbook of COVID-19 prevention and treatment. The First Affiliated Hospital, Zhejiang University School of Medicine; 2020.

31 Schönbacher M, Heinzl MW, Dauber EM, Mayr WR, Panzer S, Körmöczi GF. Granulocyte-reactive antibodies are associated with red blood cell alloimmunization. Vox Sang. 2014 Aug;107(2):200-3.

32 Triulzi DJ, Kleinman S, Kakaiya RM, Busch MP, Norris PJ, Steele WR, et al. The effect of previous pregnancy and transfusion on HLA alloimmunization in blood donors: implications for a transfusion-related acute lung injury risk reduction strategy. Transfusion. 2009 Sep;49(9):1825-35. 
33 Nguyen XD, Schulze TJ, Bugert P, LauberHärtl S, Schulz-Linkholt M, González-Schulze $\mathrm{K}$, et al. Granulocyte antibodies in male blood donors: can they trigger transfusionrelated acute lung injury? Transfusion. 2018 Aug;58(8):1894-901.

34 Keller-Stanislawski B, Reil A, Günay S, Funk $\mathrm{MB}$. Frequency and severity of transfusionrelated acute lung injury-german haemovigilance data (2006-2007). Vox Sang. 2010 Jan;98(1):70-7.

35 Chapman CE, Stainsby D, Jones H, Love E, Massey E, Win N, et al.; Serious Hazards of Transfusion Steering Group. Ten years of hemovigilance reports of transfusion-related acute lung injury in the United Kingdom and the impact of preferential use of male donor plasma. Transfusion. 2009 Mar;49(3):440-52.

36 Greinacher A, Wesche J, Hammer E, Fürll B, Völker U, Reil A, et al. Characterization of the human neutrophil alloantigen-3a. Nat Med. 2010 Jan;16(1):45-8.

37 Reil A, Wesche J, Greinacher A, Bux J. Genoand phenotyping and immunogenicity of HNA-3. Transfusion. 2011 Jan;51(1):18-24.

38 Hauck-Dlimi B, Ruppel R, Zimmermann R, Strobel J, Reil A, Eckstein R, et al. Transfusion-related alloimmune neutropenia with no pulmonary complications: one donor-five cases. Transfusion. 2016 Jan;56(1):84-90.

39 Wallis JP, Haynes S, Stark G, Green FA, Lucas GF, Chapman CE. Transfusion-related allo- immune neutropenia: an undescribed complication of blood transfusion. Lancet. 2002 Oct;360(9339):1073-4.

40 Fromont P, Prié N, Simon P, Cesbron-Gautier A, Quelvennec E, Bignon JD, et al. Granulocyte antibody screening: evaluation of a bead-based assay in comparison with classical methods. Transfusion. 2010 Dec;50(12):2643-8.

41 Bierling P, Bux J, Curtis B, Flesch B, Fung L, Lucas G, et al.; ISBT Working Party on Granulocyte Immunobiology. Recommendations of the ISBT Working Party on Granulocyte Immunobiology for leucocyte antibody screening in the investigation and prevention of antibody-mediated transfusion-related acute lung injury. Vox Sang. 2009 Apr;96(3):266-9. 